\title{
Numerical Analysis and Experimental Verification of Ti/APC-2/Kevlar Hybrid Composite Laminates Due to Low-Velocity Impact
}

\author{
Ming Hwa R. Jen, Che Kai Chang, Ying Hui Wu \\ Department of Mechanical and Electro-Mechanical Engineering, National Sun Yat-Sen University, Taiwan \\ Email: jmhr@mail.nsysu.edu.tw
}

How to cite this paper: Jen, M.H.R., Chang, C.K. and $\mathrm{Wu}$, Y.H. (2018) Numerical Analysis and Experimental Verification of Ti/APC-2/Kevlar Hybrid Composite Laminates Due to Low-Velocity Impact. Materials Sciences and Applications, 9, 1083-1095. https://doi.org/10.4236/msa.2018.913079

Received: November 14, 2018

Accepted: December 25, 2018

Published: December 28, 2018

Copyright ( $\odot 2018$ by authors and Scientific Research Publishing Inc. This work is licensed under the Creative Commons Attribution International License (CC BY 4.0).

http://creativecommons.org/licenses/by/4.0/ (c) (i) Open Access

\begin{abstract}
The mechanical properties of Ti/APC-2/Kevlar/epoxy hybrid composite laminates after low velocity impact were investigated at room temperature. There were three types of samples, including three layered $\left[\mathrm{Ti} /(0 / 90)_{s} / \mathrm{Ti}\right]$, five layered $\left[\mathrm{Ti} /(0 / 90)_{2} / \overline{\mathrm{Ti}}\right]_{\mathrm{s}}$ and nine layered $\left[\mathrm{Ti} / \operatorname{Kevlar} / \mathrm{Ti} /(0 / 90)_{2} / \overline{\mathrm{Ti}}\right]_{s}$. The lay-ups of APC-2 were cross-ply, while Ti layers were treated by chromic acid anodic method. Ti and APC-2 were stacked to fabricate the composite laminates via hot press curing process. Kevlar layers were added to fabricate nine-layered composite laminates via vacuum assisted resin transfer molding. The drop-weight tests were conducted with a hemispherical nosed projectile in $10 \mathrm{~mm}$ diameter. The impact loads were $5 \mathrm{~kg}$ and $10 \mathrm{~kg}$ and impact heights were adjusted to penetrate samples or the maximum height $1.50 \mathrm{~m}$. The static tensile tests were conducted to measure the residual mechanical properties after impact. The free body drop tests were also simulated by using finite element method and software ANSYS LS-DYNA3D. The results showed that the bottom Ti layer absorbed more internal energy than the top Ti layer, then the cracks were found in the bottom Ti layer more often. The ultimate tensile strength reduced significantly after impact. The initial longitudinal compliance increased with the impact height increasing and decreased after the samples penetrated. Comparing the experimental data with the numerical results, it was found that the damage of the latter was more serious than that of the former. On the conservative side, the results of numerical simulation are acceptable and adopted for applications when no testing data available.
\end{abstract}

\section{Keywords}

Titanium, APC-2, Kevlar, Low-Velocity, Impact, Tensile Test 


\section{Introduction}

Fiber metal laminates (FMLs) are hybrid composite structures constructed from thin sheets of metal alloys and plies of fiber-reinforced polymeric materials. The first FMLs, called aramid-reinforced aluminum laminates (ARALLs), were introduced in 1978 at the Faculty of Aerospace Engineering at Delft University of Technology in the Netherlands [1]. In 1990, an improved type of ARALL called glass laminate aluminum reinforced epoxy (GLARE), or ARALL with glass fibers, was successfully developed [2]. Furthermore, Lin et al. [3] developed carbon-reinforced aluminum laminates, which contain carbon fibers (CFs) rather than aramid fibers. FMLs have the advantages of metal alloys and fiber-reinforced plastic (FRP) composites. Castrodeza et al. [4] demonstrated that GLARE and ARALL possess superior fracture toughness and crack tolerance to those of their constituent alloys. Vlot [5] [6] investigated GLARE and ARALL, finding that the impact resistance of FMLs was superior to that of the studied FRPs. Gunnink [7] showed that ARALL retains excellent durability even after very long exposure to highly aggressive environments. Additionally, FMLs containing various types of metal alloys and FRPs have been developed by researchers for wider applications. Khalili et al. [8] studied the mechanical properties of steel-aluminum-FRP laminates. Furthermore, Zhou et al. [9] investigated the tensile behavior of Kevlar fiber-reinforced aluminum laminates. Jen et al. developed magnesium/CF/PEEK nanocomposite laminates [10] as well as titanium (Ti)/CF/PEEK nanocomposite laminates [11] and obtained their mechanical properties at elevated temperatures.

Owing to the brittleness of thermosetting matrix resulted by cross-linking, the epoxy resins and epoxy-based fiber composites are susceptible to impact damage. Thermoplastics, having greater toughness, are considered to be potential for alleviating this problem [12]. Although damage inflicted by low-velocity impact appears quite complicated, the major failure modes include only matrix cracking, delamination, and fiber breakage [13]. The delamination mode of failure is induced by matrix cracks which occur prior to other failure modes. Thus, the suppression of matrix cracking will suppress delamination. It is conceivable that the use of tougher matrices will yield composites that are more resistant to impact damage.

Except for the degree of damage, the plate specimens did not differ from beam specimens in failure modes or impact tolerance properties [14], i.e., no plate size effect. The post impact load-carrying capability of a composite laminate is of prime concern to the design engineer. After a tool-drop type accident where no damage is visible from the surface, the structure is still expected to carry the full spectrum of loading. However, it may be wrong of overestimation. In all cases the residual strength decreased as the impact velocity increased. From the results [15] the tough matrix composites may provide excellent impact resistance properties at low-impact velocities. However, beyond a certain threshold velocity, i.e., $\mathrm{v} \geq 25 \mathrm{~m} / \mathrm{s}$, the use of tough matrix materials may result in more laminate tensile 
and flexural strength reduction than that of brittle matrix materials. Additionally, the PEEK composites have significantly lower contact rigidity, i.e., for a given contact force, the resulting indentation in the PEEK composites would be larger, yielding a larger contact area, and, therefore, a low contact pressure. A larger contact area with lower pressure will reduce the transverse shear stress concentration and thus minimize local matrix cracking.

Anodic method is a commonly used surface treatment, however, the bonding capability of polymer composites to titanium thin plates is still a problem. In order to improve the interfacial bonding capability, Ramani et al. [16] found the chromic acid anodic method was excellent. Chromic acid anodic oxidation produced an oxide layer of thickness $40-80 \mathrm{~nm}$ for the $5 \mathrm{~V}$ and $10 \mathrm{~V}$ treatments [17]. In recent years, inorganic nanoparticles filled polymer composites have attracted attention because the filler/matrix interface in these composites might constitute a great area and influence the properties of composites at rather low filler concentration [18].

Based on above-mentioned statements, the Ti/APC-2 FLMs were fabricated to investigate their resistance to impact loads by semi-sphere bullet head, measure residual mechanical properties and compare the data with the results of numerical simulation by using ANSYS software LS DYNA-3D.

\section{Specimen Fabrication}

The twelve-inch wide prepregs of Carbon/PEEK (Cytec Industries Inc., USA) unidirectional plies were cut and stacked into cross-ply [0/90] laminates. The grade 1 (H: $0.015 \%$, O: $0.18 \%$, N: $0.03 \%$, Fe: $0.2 \%$, C: $0.08 \%)$ Ti sheets, supplied by Kobe Steel Ltd (Japan), were $0.5 \mathrm{~mm}$ thick after rolled, heated and flattened with scratch brushing. The ultimate tensile strength of Ti is $353 \mathrm{MPa}$, and modulus of elasticity $109 \mathrm{GPa}$.

After a series of tests, the surface treatment by chromic acid anodic method of electro-plating was found better as demonstrated by the results of tensile tests. The anodic oxide coating film was observed uniform by Scanning Electron Microscope, and the composition of coating consisting of $\mathrm{TiO}_{2}$ by Energy-Dispersive $\mathrm{X}$-ray Spectroscopy.

The APC-2 prepregs were sandwiched with the Ti alloy sheets to produce Ti/APC-2 hybrid $\left[\mathrm{Ti} /(0 / 90)_{s} / \mathrm{Ti}\right]$ three-layered and $\left[\mathrm{Ti} /(0 / 90)_{2} / \overline{\mathrm{Ti}}\right]_{\mathrm{s}}$ five-layered laminated composites. The hot press and modified diaphragm curing process were adopted to fabricate laminates [19]. The hybrid composite specimen was a rectangular plate of $240 \mathrm{~mm}(\mathrm{~L}) \times 25 \mathrm{~mm}(\mathrm{~W})$ with thickness $1.55 \mathrm{~mm}$ and 2.50 $\mathrm{mm}$. Additionally, the $4.50 \mathrm{~mm}$ thick $\left[\mathrm{Ti} / \mathrm{Kevlar} / \mathrm{Ti} /(0 / 90)_{2} / \overline{\mathrm{Ti}}\right]_{\mathrm{s}}$ nine-layered laminates were covered by Kevlar layers via vacuum assisted resin transfer molding.

\section{Numerical Analysis}

In simulation the same sample geometry, dimensions and materials as fabricated 
were adopted such as $\left[\mathrm{Ti} /(0 / 90)_{s} / \mathrm{Ti}\right],\left[\mathrm{Ti}(0 / 90)_{2} / \overline{\mathrm{Ti}}\right]_{s}$ and $\left[\mathrm{Ti} / \mathrm{Kevlar} / \mathrm{Ti} /(0 / 90)_{2} /\right.$ $\overline{\mathrm{Ti}}]_{\mathrm{s}}$ three hybrid composite laminates. The finite element analysis and ANSYS/LS-DYNA 3D software were used to simulate the impact whole process starting from the contact and penetration of top layer step by step down to the full penetration of bottom layer with the zero velocity left. Due to the symmetry only one quarter of a sample was considered. The 3D Solid 164 elements with eight nodes and nine degrees of freedom for each node were used to construct the model. The bullet was a semispherical nosed projectile of $10 \mathrm{~mm}$ diameters. The assumed boundary conditions were that no displacement along the symmetry edges, totally constrained for other two free edges and a plastic cushion placed at the bottom of laminate to avoid rebounce. The failure criterion was based on the value of principal strain. Herein, the failure values of principal strains were 0.0088 for $90^{\circ}$ Carbon fibers, 0.189 for Ti alloy and 0.08 for Kevlar fibers, respectively.

Based on the received results as valuable references we setup impact instruments for the impact tests and compared the experimental data and failure mechanisms with the numerical results for comparison.

\section{Experimental Work}

The free drop impact testing systems were self-constructed as shown in Figure 1. The impactors with a semi-spherical nosed projectile were $5 \mathrm{~kg}$ and $10 \mathrm{~kg}$ of weight respectively. Three types of samples were impacted by two weights, $5 \mathrm{~kg}$ and $10 \mathrm{~kg}$ at different height. All the samples were cut into $12 \mathrm{~cm}(\mathrm{~L}) \times 1.25 \mathrm{~cm}$ (W) rectangles by using the electrical discharge method. The samples were placed at the top of a thick rubber plate and fixed at the bottom of the impact system to be impacted one by one. An MTS- 810 servohydraulic computer-controlled dynamic material testing machine was used to conduct the tensile

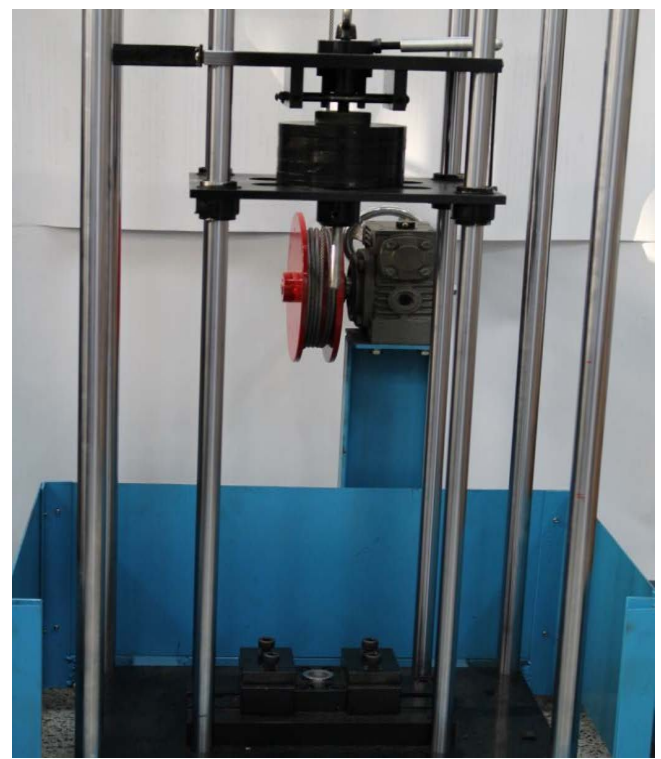

Figure 1. The free drop impact testing system. 
tests after the free drop impact. Then, the impacted samples were due to tensile tests to obtain their mechanical properties.

\section{Results}

The numerical simulation results of velocity, impact energy, height and failure mechanisms of $\left[\mathrm{Ti} /(0 / 90)_{s} / \mathrm{Ti}\right]_{s}$ three-layered laminates were listed in Table 1. The data of three-layered laminates due to $5 \mathrm{~kg}$ free drop tests were listed in Table 1 for contrast. The numerical results and the data due to $10 \mathrm{~kg}$ free drop tests were listed in Table 2 together. The numerical results and test data of $\left[\mathrm{Ti} /(0 / 90)_{2} /\right.$ $\overline{\mathrm{Ti}}]_{\mathrm{s}}$ five-layered laminates subjected to $5 \mathrm{~kg}$ free drop were tabulated in Table 3

Table 1. The numerical results and impact damage mechanisms of three-layered samples due to $5 \mathrm{~kg}$ free drop tests.

\begin{tabular}{|c|c|c|c|c|c|}
\hline $\begin{array}{l}\text { Velocity } \\
(\mathrm{m} / \mathrm{s})\end{array}$ & $\begin{array}{l}\text { Energy } \\
\quad(\mathrm{J})\end{array}$ & $\begin{array}{c}\text { Height of } \\
\text { simulation }(\mathrm{m})\end{array}$ & $\begin{array}{l}\text { Height of } \\
\text { impact }(\mathrm{m})\end{array}$ & $\begin{array}{l}\text { Damage model of } \\
\text { simulation }\end{array}$ & $\begin{array}{c}\text { Damage mechanisms } \\
\text { of impact test }\end{array}$ \\
\hline 2.34 & 13.69 & 0.28 & 0.49 & $\begin{array}{l}\text { APC- } 2: \mathrm{f} \\
\text { Ti: } 1^{\text {st }}, \mathrm{f}\end{array}$ & $\begin{array}{c}\text { Ti: } 1^{\text {st }}, \mathrm{d} ; 2^{\text {nd }}, \mathrm{f} \\
\text { depression: } 5.79 \mathrm{~mm}\end{array}$ \\
\hline 2.43 & 14.76 & 0.30 & 0.53 & $\begin{array}{c}\text { APC- } 2: \mathrm{f} \\
\mathrm{Ti}: 1^{\text {st }} \text { and } 2^{\text {nd }}, \mathrm{f}\end{array}$ & - \\
\hline 2.47 & 15.25 & 0.31 & 0.55 & $\begin{array}{c}\text { APC- } 2: \mathrm{f} \\
\text { Ti: } 1^{\text {st }} \text { and } 2^{\text {nd }}, f\end{array}$ & $\begin{array}{c}\mathrm{Ti}: 1^{\text {st }}, \mathrm{d} ; 2^{\text {nd }}, \mathrm{f} ; \\
\text { depression: } 6.40 \mathrm{~mm}\end{array}$ \\
\hline 2.51 & 15.75 & 0.32 & 0.57 & ballistic limit & - \\
\hline 2.62 & 17.16 & 0.35 & 0.62 & $\mathrm{p}$ & $\begin{array}{c}\mathrm{Ti}: 1^{\text {st }}, \mathrm{d} ; 2^{\text {nd }}, \mathrm{f} ; \\
\text { depression: } 6.71 \mathrm{~mm}\end{array}$ \\
\hline 2.80 & 19.6 & 0.40 & 0.71 & $\mathrm{p}$ & $\begin{array}{c}\text { Ti: } 1^{\text {st }}, \mathrm{f} ; 2^{\text {nd }}, \mathrm{f} ; \\
\text { depression: } 7.02 \mathrm{~mm}\end{array}$ \\
\hline 2.97 & 22.05 & 0.45 & 0.79 & $\mathrm{p}$ & $\begin{array}{c}\text { Ti: } 1^{\text {st }}, \mathrm{f} ; 2^{\text {nd }}, \mathrm{f} ; \\
\text { depression: } 7.72 \mathrm{~mm}\end{array}$ \\
\hline 3.13 & 24.49 & 0.50 & 0.88 & $\mathrm{p}$ & $\begin{array}{c}\text { Ti: } 1^{\text {st }}, \mathrm{f} ; 2^{\text {nd }}, \mathrm{f} ; \\
\text { depression: } 8.04 \mathrm{~mm}\end{array}$ \\
\hline 3.28 & 26.90 & 0.55 & 0.97 & $\mathrm{p}$ & $\begin{array}{c}\text { Two samples near } \\
\text { penetration, one penetrated; } \\
\text { depression: } 9.30 \mathrm{~mm}\end{array}$ \\
\hline 3.34 & 27.89 & 0.57 & 1.01 & $\mathrm{p}$ & $\mathrm{p}$ \\
\hline
\end{tabular}

Notes: $1^{\text {st }}$ denotes the first layer; $2^{\text {nd }}$ denotes the second layer; $d$ : depressed; $f$ : fractured; p: penetration; APC-2: APC-2 laminates; Ti: Ti sheet; -: not available.

Table 2. The numerical results and impact damage mechanisms of three-layered samples due to $10 \mathrm{~kg}$ free drop tests.

\begin{tabular}{cccccc}
\hline $\begin{array}{c}\text { Velocity } \\
(\mathrm{m} / \mathrm{s})\end{array}$ & $\begin{array}{c}\text { Energy } \\
(\mathrm{J})\end{array}$ & $\begin{array}{c}\text { Height of } \\
\text { simulation }(\mathrm{m})\end{array}$ & $\begin{array}{c}\text { Height of } \\
\text { impact }(\mathrm{m})\end{array}$ & $\begin{array}{c}\text { Damage model of } \\
\text { simulation }\end{array}$ & $\begin{array}{c}\text { Damage mechanisms } \\
\text { of impact test }\end{array}$ \\
\hline 1.4 & 9.80 & 0.10 & 0.18 & $\begin{array}{c}\text { APC-2: } \mathrm{f} \\
\text { Ti: } 1^{\text {st }} \text { and } 2^{\text {nd }}, \mathrm{f}\end{array}$ & Ti: d; depression: $5.01 \mathrm{~mm}$ \\
1.72 & 14.79 & 0.15 & 0.27 & $\begin{array}{c}\text { APC-2: } \mathrm{f} \\
\text { Ti: } 1^{\text {st }} \text { and } 2^{\text {nd }}, \mathrm{f}\end{array}$ & $\begin{array}{c}\text { Ti: Ti: } 1^{\text {st }}, \mathrm{d} ; 2^{\text {nd }}, \mathrm{f} ; \\
\text { depression: } 6.13 \mathrm{~mm}\end{array}$ \\
1.77 & 15.66 & 0.16 & 0.28 & ballistic limit & - \\
\hline
\end{tabular}




\section{Continued}

\begin{tabular}{cccccc}
\hline 1.98 & 19.60 & 0.20 & 0.35 & $\mathrm{p}$ & $\begin{array}{c}\text { Ti: Ti: } 1^{\text {st }} \text { and } 2^{\text {nd }}, \mathrm{f} ; \\
\text { depression: } 7.41 \mathrm{~mm}\end{array}$ \\
2.21 & 24.42 & 0.25 & 0.44 & $\mathrm{p}$ & $\mathrm{p}$ \\
2.34 & 27.38 & 0.28 & 0.49 & $\mathrm{p}$ & $\mathrm{p}$ \\
\hline
\end{tabular}

Notes: $1^{\text {st }}$ denotes the first layer; $2^{\text {nd }}$ denotes the second layer; $d$ : depressed; $f$ : fractured; p: penetration; APC-2: APC-2 laminates; Ti: Ti sheet; -: not available.

Table 3. The numerical results and impact damage mechanisms of five-layered samples due to $5 \mathrm{~kg}$ free drop tests.

\begin{tabular}{|c|c|c|c|c|c|}
\hline $\begin{array}{l}\text { Velocity } \\
(\mathrm{m} / \mathrm{s})\end{array}$ & $\begin{array}{l}\text { Energy } \\
\quad(\mathrm{J})\end{array}$ & $\begin{array}{c}\text { Height of } \\
\text { simulation }(\mathrm{m})\end{array}$ & $\begin{array}{l}\text { Height of } \\
\text { impact }(\mathrm{m})\end{array}$ & $\begin{array}{c}\text { Damage model of } \\
\text { simulation }\end{array}$ & $\begin{array}{c}\text { Damage mechanisms } \\
\text { of impact test }\end{array}$ \\
\hline 2.43 & 14.76 & 0.30 & 0.53 & $\begin{array}{l}\text { APC-2: } \mathrm{f} \\
\text { Ti: } 1^{\text {st }}, \mathrm{f}\end{array}$ & Ti: d; depression: $5.11 \mathrm{~mm}$ \\
\hline 2.62 & 17.16 & 0.35 & 0.62 & $\begin{array}{l}\text { APC-2: } \mathrm{f} \\
\text { Ti: } 1^{\text {st }}, \mathrm{f}\end{array}$ & - \\
\hline 2.80 & 19.60 & 0.40 & 0.71 & $\begin{array}{l}\text { APC- } 2: \mathrm{f} \\
\text { Ti: } 1^{\text {st }}, \mathrm{f}\end{array}$ & Ti: $3^{\text {rd }}, \mathrm{f}$; depression: $5.78 \mathrm{~mm}$ \\
\hline 2.97 & 22.05 & 0.45 & 0.79 & $\begin{array}{c}\text { APC- } 2: f \\
\text { Ti: } 1^{\text {st }} \text { and } 2^{\text {nd }} f\end{array}$ & - \\
\hline 3.13 & 24.49 & 0.50 & 0.88 & $\begin{array}{c}\text { APC- } 2: \mathrm{f} \\
\text { Ti: } 1^{\text {st }}, 2^{\text {nd }} \text { and } 3^{\text {rd }}, f\end{array}$ & Ti: $3^{\text {rd }}$, f; depression: $6.22 \mathrm{~mm}$ \\
\hline 3.19 & 25.44 & 0.52 & 0.92 & ballistic limit & Ti: $3^{\text {rd }}, \mathrm{f} ;$ depression: $6.52 \mathrm{~mm}$ \\
\hline 3.43 & 29.41 & 0.6 & 1.06 & $\mathrm{p}$ & Ti: $3^{\text {rd }}$, f; depression: $6.96 \mathrm{~mm}$ \\
\hline 3.57 & 31.86 & 0.65 & 1.15 & $\mathrm{p}$ & $\begin{array}{c}\text { Ti: } 1^{\text {st }} \text { and } 3^{\text {rd }}, \mathrm{f} \\
\text { depression: } 7.20 \mathrm{~mm}\end{array}$ \\
\hline 3.71 & 34.41 & 0.70 & 1.24 & $\mathrm{p}$ & $\begin{array}{c}\text { Ti: } 1^{\text {st }} \text { and } 3^{\text {rd }}, \mathrm{f} \\
\text { depression: } 7.63 \mathrm{~mm}\end{array}$ \\
\hline 3.84 & 36.86 & 0.75 & 1.33 & $\mathrm{p}$ & $\begin{array}{c}\text { Ti: } 1^{\text {st }} \text { and } 3^{\text {rd }}, \mathrm{f} \\
\text { depression: } 7.85 \mathrm{~mm}\end{array}$ \\
\hline 3.96 & 39.20 & 0.80 & 1.41 & $\mathrm{p}$ & $\begin{array}{c}\text { Ti: } 1^{\text {st }} \text { and } 3^{\text {rd }}, \mathrm{f} \\
\text { depression: } 8.00 \mathrm{~mm}\end{array}$ \\
\hline 4.08 & 41.62 & 0.85 & 1.50 & $\mathrm{p}$ & $\begin{array}{c}\text { Ti: } 1^{\text {st }} \text { and } 3^{\text {rd }}, \mathrm{f} \\
\text { depression: } 9.10 \mathrm{~mm}\end{array}$ \\
\hline
\end{tabular}

Notes: $1^{\text {st }}$ denotes the first layer; $2^{\text {nd }}$ denotes the second layer; $3^{\text {rd }}$ denotes the third layer; $d$ : depressed; $f$ : fractured; p: penetration; APC-2: APC-2 laminates; Ti: Ti sheet; -: not available.

and due to $10 \mathrm{~kg}$ free drop were listed in Table 4, respectively. The results and data of $\left[\mathrm{Ti} / \mathrm{Kev} / \mathrm{Ti}(0 / 90)_{2} / \overline{\mathrm{Ti}}\right]_{\mathrm{s}}$ nine-layered laminates due to $10 \mathrm{~kg}$ free drop were tabulated in Table 5 .

The simulation picture of $5 \mathrm{~kg}$ free drop at different height impacted onto the five-layered laminates was shown in Figure 2. The pictures of impact damage due to $5 \mathrm{~kg}$ free drop at height $1.5 \mathrm{~m}$ onto five-layered laminates were shown in Figure 3 for example. The relationships of load and initial compliance vs. the height of free drop were plotted in Figure 4 for example. 
Table 4. The numerical results and impact damage mechanisms of five-layered samples due to $10 \mathrm{~kg}$ free drop tests.

\begin{tabular}{|c|c|c|c|c|c|}
\hline $\begin{array}{l}\text { Velocity } \\
(\mathrm{m} / \mathrm{s})\end{array}$ & $\begin{array}{l}\text { Energy } \\
\quad(\mathrm{J})\end{array}$ & $\begin{array}{c}\text { Height of } \\
\text { simulation }(\mathrm{m})\end{array}$ & $\begin{array}{l}\text { Height of } \\
\text { impact }(\mathrm{m})\end{array}$ & $\begin{array}{l}\text { Damage model of } \\
\text { simulation }\end{array}$ & $\begin{array}{c}\text { Damage mechanisms } \\
\text { of impact test }\end{array}$ \\
\hline 1.40 & 9.80 & 0.10 & 0.18 & $\begin{array}{l}\text { APC-2:f } \\
\text { Ti: } 1^{\text {st }}, f\end{array}$ & Ti: d; depression: $4.72 \mathrm{~mm}$ \\
\hline 1.98 & 19.60 & 0.20 & 0.35 & $\begin{array}{c}\text { APC-2: } \mathrm{f} \\
\mathrm{Ti}: 1^{\text {st }} \text { and } 2^{\text {nd }}, \mathrm{f}\end{array}$ & $\begin{array}{c}\mathrm{Ti}: 1^{\mathrm{st}}, \mathrm{d} ; 3^{\mathrm{rd}}, \mathrm{f} \\
\text { depression: } 6.09 \mathrm{~mm}\end{array}$ \\
\hline 2.21 & 24.42 & 0.25 & 0.44 & $\begin{array}{l}\text { APC-2: } \mathrm{f} \\
\text { Ti: } 1^{\text {st }}, \mathrm{f}\end{array}$ & - \\
\hline 2.30 & 26.45 & 0.27 & 0.48 & ballistic limit & - \\
\hline 2.34 & 27.38 & 0.28 & 0.49 & $\mathrm{p}$ & - \\
\hline 2.43 & 29.52 & 0.30 & 0.53 & $\mathrm{p}$ & $\begin{array}{c}\text { Ti: } 1^{\text {st }}, \mathrm{d} ; 3^{\text {rd }}, \mathrm{f} ; \\
\text { depression: } 7.44 \mathrm{~mm}\end{array}$ \\
\hline 2.80 & 39.20 & 0.40 & 0.71 & $\mathrm{p}$ & $\begin{array}{l}\text { Ti: } 1^{\text {st }}, \mathrm{d} ; 2^{\text {nd }} \text { and } 3^{\text {rd }}, \mathrm{f} \\
\text { depression: } 8.70 \mathrm{~mm}\end{array}$ \\
\hline 3.13 & 48.98 & 0.50 & 0.88 & $\mathrm{p}$ & $\begin{array}{l}\text { Ti: } 1^{\text {st }}, 2^{\text {nd }} \text { and } 3^{\text {rd }}, \mathrm{f} \\
\text { depression: } 9.56 \mathrm{~mm}\end{array}$ \\
\hline 3.28 & 53.79 & 0.55 & 0.97 & $\mathrm{p}$ & $\begin{array}{c}\text { Near penetration; } \\
\text { depression: } 10.68 \mathrm{~mm}\end{array}$ \\
\hline 3.43 & 58.82 & 0.60 & 1.06 & $\mathrm{p}$ & $\mathrm{p}$ \\
\hline
\end{tabular}

Notes: $1^{\text {st }}$ denotes the first layer; $2^{\text {nd }}$ denotes the second layer; $3^{\text {rd }}$ denotes the third layer; d: depressed; $f$ : fractured; p: penetration; APC-2: APC-2 laminates; Ti: Ti sheet; -: not available.

Table 5. The numerical results and impact damage mechanisms of nine-layered samples due to $10 \mathrm{~kg}$ free drop tests.

\begin{tabular}{|c|c|c|c|c|c|}
\hline $\begin{array}{l}\text { Velocity } \\
(\mathrm{m} / \mathrm{s})\end{array}$ & $\begin{array}{l}\text { Energy } \\
\quad(J)\end{array}$ & $\begin{array}{c}\text { Height of } \\
\text { simulation }(\mathrm{m})\end{array}$ & $\begin{array}{l}\text { Height of } \\
\text { impact }(\mathrm{m})\end{array}$ & $\begin{array}{c}\text { Damage model of } \\
\text { simulation }\end{array}$ & $\begin{array}{c}\text { Damage mechanisms } \\
\text { of impact test }\end{array}$ \\
\hline 2.43 & 29.52 & 0.30 & 0.53 & $\begin{array}{l}\text { APC-2: f; Kevlar: } \mathrm{f} \\
\text { Ti: } 1^{\text {st }}, \mathrm{f}\end{array}$ & - \\
\hline 2.62 & 34.32 & 0.35 & 0.62 & $\begin{array}{l}\text { APC-2: f; Kevlar: f; } \\
\text { Ti: } 1^{\text {st }} 5^{\text {th }}, f\end{array}$ & - \\
\hline 2.80 & 39.2 & 0.40 & 0.71 & $\begin{array}{l}\text { APC-2: f; Kevlar: f; } \\
\text { Ti } 1^{\text {st }}, 2^{\text {nd }} \text { and } 5^{\text {th }}, f\end{array}$ & $\begin{array}{l}\mathrm{Ti}, \mathrm{APC}-2 \text {, and Kevlar, } \mathrm{d} \text {; } \\
\text { depression: } 8.13 \mathrm{~mm}\end{array}$ \\
\hline 2.87 & 41.18 & 0.42 & 0.74 & $\begin{array}{l}\text { APC-2: } f \text {; Kevlar: } f \\
\text { Ti: } 1^{\text {st }}, 2^{\text {nd }} \text { and } 5^{\text {th }}, \mathrm{f}\end{array}$ & - \\
\hline 2.97 & 44.1 & 0.45 & 0.79 & $\begin{array}{l}\text { APC-2: f; Kevlar: } f \\
\text { Ti: } 1^{\text {st }}, 2^{\text {nd }}, 4^{\text {th }} \text { and } 5^{\text {th }}, f\end{array}$ & $f$ \\
\hline 3.13 & 48.98 & 0.50 & 0.88 & $\begin{array}{c}\text { APC-2: f; Kevlar: f; } \\
\text { Ti: } \mathrm{f}\end{array}$ & $\begin{array}{c}\text { Ti: } 1^{\text {st }}-4^{\text {th }}, \text { APC- } 2 \text {, and Kevlar, } \\
\text { d; Ti: } 5^{\text {th }}, \text { f; depression: } 8.13 \\
\text { mm }\end{array}$ \\
\hline 3.22 & 51.84 & 0.53 & 0.93 & ballistic limit & - \\
\hline 3.28 & 53.79 & 0.55 & 0.97 & $\mathrm{p}$ & - \\
\hline 3.43 & 58.82 & 0.60 & 1.06 & $\mathrm{p}$ & $\begin{array}{c}\text { Ti: } 2^{\text {nd }}-4^{\text {th }}, \text { APC- } 2 \text {, and Kevlar, } \\
\text { d; Ti: } 1^{\text {st }} \text { and } 5^{\text {th }} \text {, f; depression: } \\
9.68 \mathrm{~mm}\end{array}$ \\
\hline
\end{tabular}




\section{Continued}

\begin{tabular}{|c|c|c|c|c|c|}
\hline 3.71 & 68.82 & 0.70 & 1.24 & $\mathrm{p}$ & $\begin{array}{c}\text { Ti: } 2^{\text {nd }}-4^{\text {th }}, \text { APC- } 2 \text {, and Kevlar, } \\
\text { d; Ti: } 1^{\text {st }} \text { and } 5^{\text {th }}, \text { f; depression: } \\
10.61 \mathrm{~mm}\end{array}$ \\
\hline 4.08 & 82.23 & 0.85 & 1.50 & $\mathrm{p}$ & $\begin{array}{c}\text { Ti: } 2^{\text {nd }}-4^{\text {th }}, \text { APC- } 2 \text {, and Kevlar, } \\
\text { d; Ti: } 1^{\text {st }} \text { and } 5^{\text {th }}, \text { f; depression: } \\
11.61 \mathrm{~mm}\end{array}$ \\
\hline
\end{tabular}

Notes: $1^{\text {st }}$ denotes the first layer; $2^{\text {nd }}$ denotes the second layer; $4^{\text {th }}$ denotes the fourth layer; $5^{\text {th }}$ denotes the fifth layer; d: depressed; f: fractured; p: penetration; APC-2: APC-2 laminates; Ti: Ti sheet; -: not available.

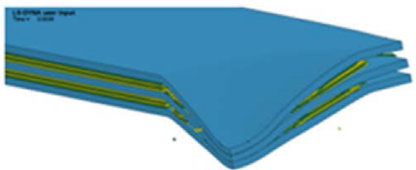

(a)

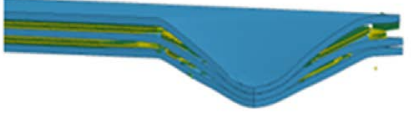

(b)

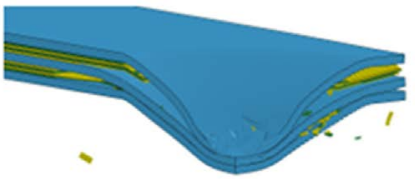

(c)

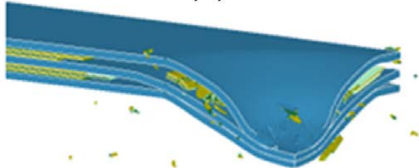

(d)

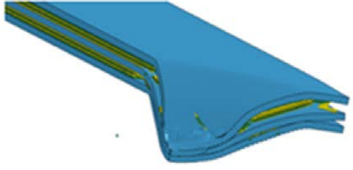

(e)

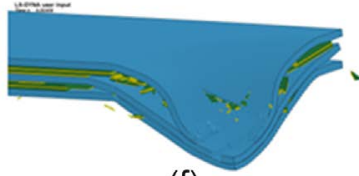

(f)

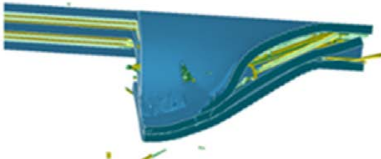

(g)

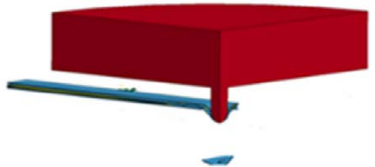

(h)

Figure 2. Damage simulation pictures of five-layered laminates due to $5 \mathrm{~kg}$ free drop impact at heights (a) $0.2 \mathrm{~m}$ (b) $0.25 \mathrm{~m} \mathrm{(c)} 0.3 \mathrm{~m}$ (d) $0.35 \mathrm{~m} \mathrm{(e)} 0.4 \mathrm{~m}$ (f) $0.45 \mathrm{~m} \mathrm{(g)} 0.5 \mathrm{~m} \mathrm{(h)}$ scheme of impact.

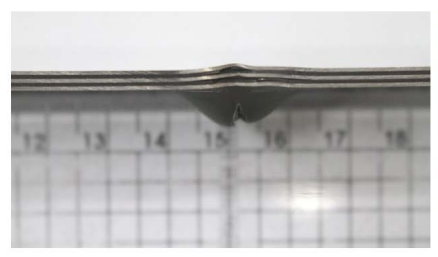

(a)

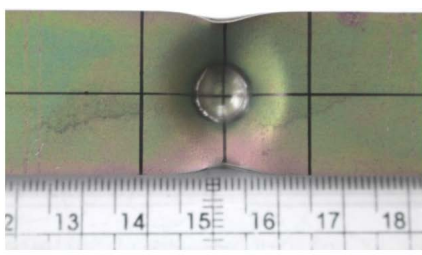

(b)

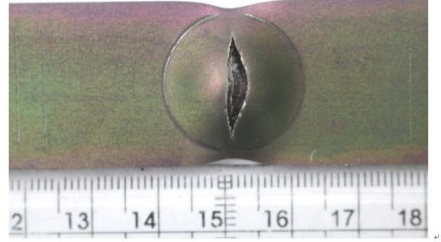

(c)

Figure 3. The photos of impact damage on five-layered laminates due to $5 \mathrm{~kg}$ free drop at $1.5 \mathrm{~m}$ high (a) side view (b) top view (c) bottom view. 


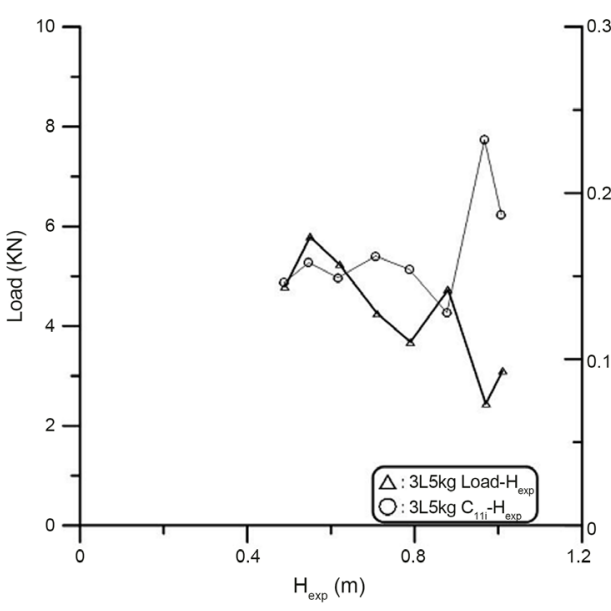

(a)

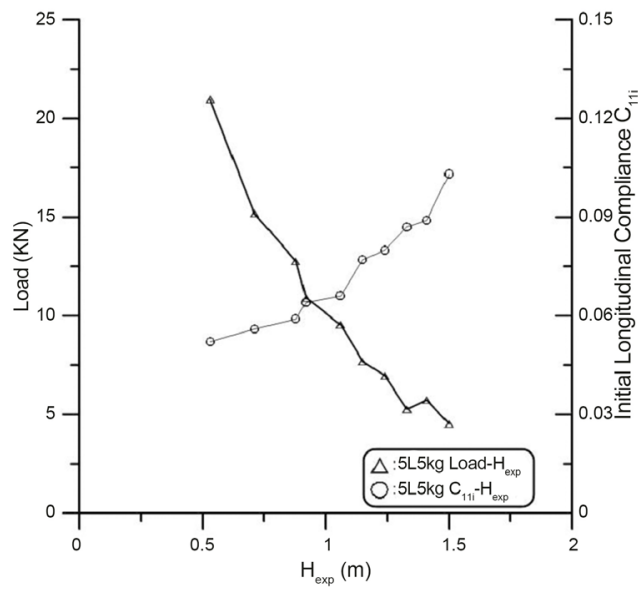

(c)

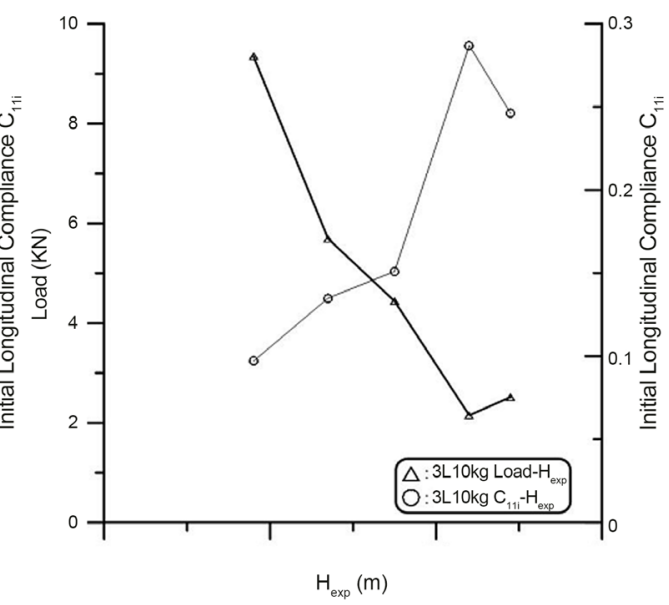

(b)

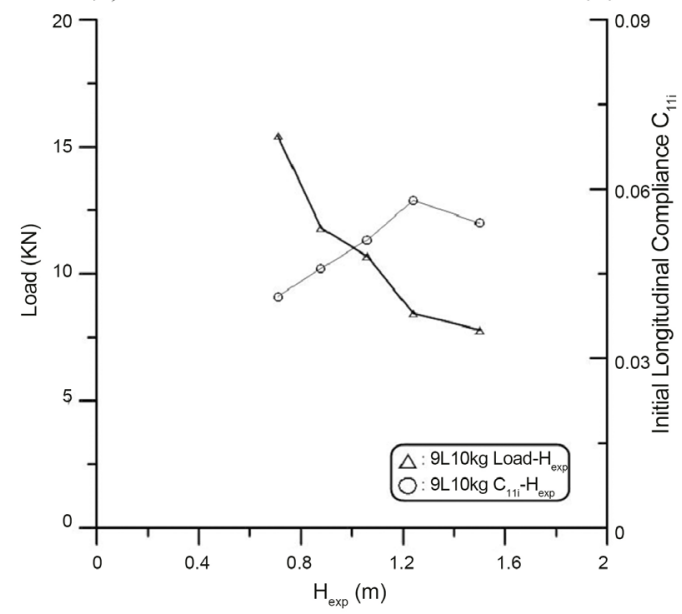

(e)

Figure 4. The empirical results of load, initial longitudinal compliance and drop height of free drop tests for (a) three-layered samples due to $5 \mathrm{~kg}$ (b) three-layered samples due to $10 \mathrm{~kg}$ (c) five-layered samples due to $5 \mathrm{~kg}$ (d) five-layered samples due to $10 \mathrm{~kg}$ (e) nine-layered samples due to $10 \mathrm{~kg}$.

\section{Discussion}

The numerical simulation by LS DYNA-3D software and finite element method 
was adopted first to obtain the results as valuable information and references for the next step impact tests. Inversely, the test data provided an important contrast in comparison with the numerical results. It was also found that the used software simulation was acceptably feasible without the impact tests because the results were more serious damage than those of test data. Thus, the numerical results can be used on the conservation side in case of testing data not available.

Due to the limited space of our lab the free drop impact tests were adopted alternatively. As can be seen in Tables 1-5 the real heights of free drop were much higher than those of predicted heights by simulation. It was mainly attributed to the friction in free drop of the testing system. From the impactor drop weight and height associated with measured time of starting impact we calculated the velocity of impact for numerical simulation. To keep the same impact energy acting on the samples the heights adjusted and elevated were necessarily needed. All the three types of samples after free drop impact tests were subjected to tensile tests and the depression pictures were illustrated in Figure 5. The general trend was that the applied loads decreased with the increasing height, however, the initial compliances of damage samples increased with the increasing height inversely.

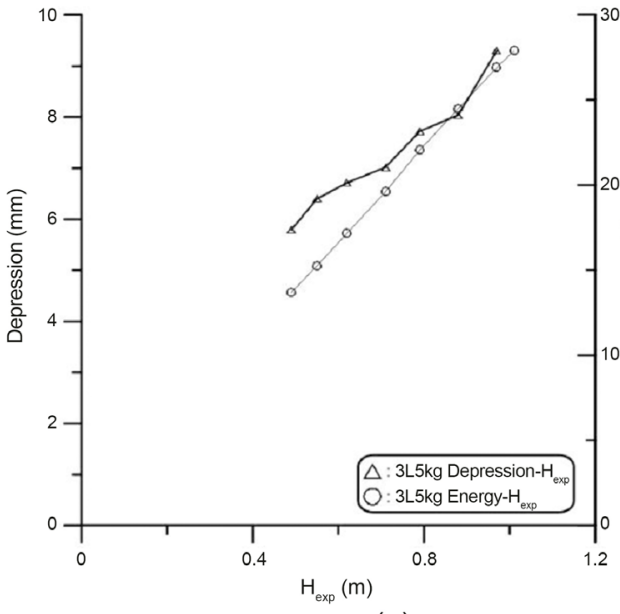

(a)

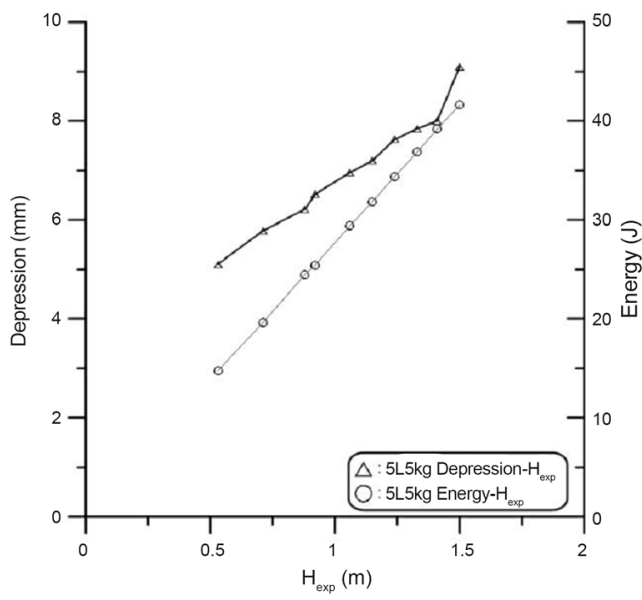

(c)

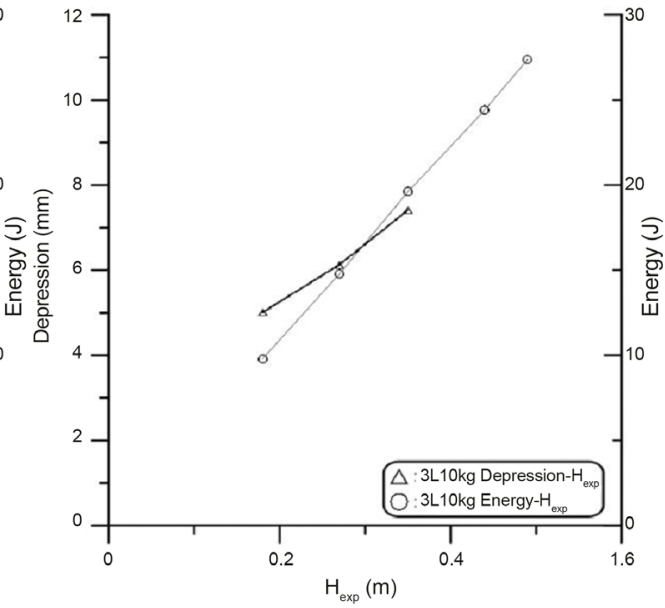

(b)

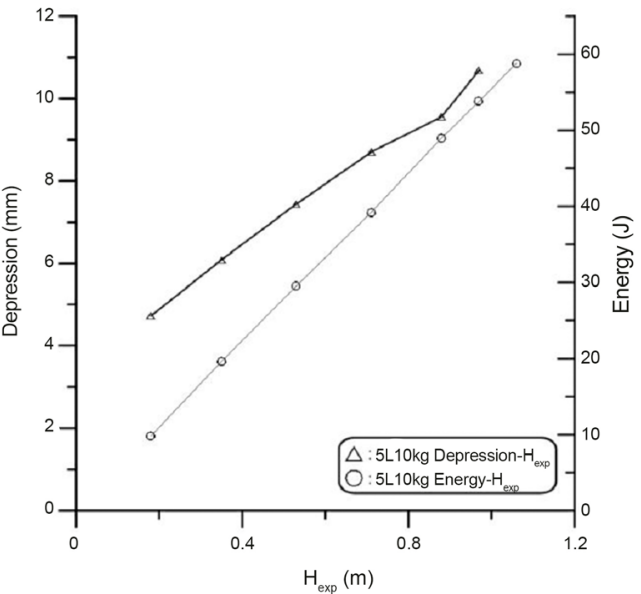

(d) 


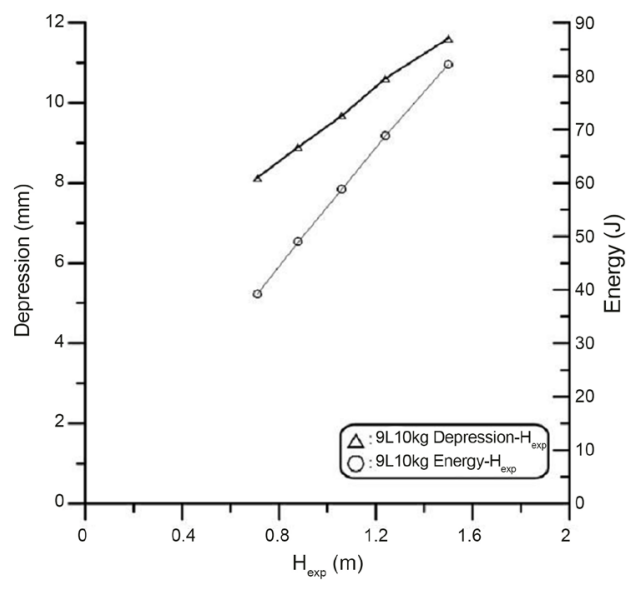

(e)

Figure 5. The empirical results of depression, energy and drop height of free drop tests for (a) three-layered samples due to $5 \mathrm{~kg}$ (b) three-layered samples due to $10 \mathrm{~kg}$ (c) five-layered samples due to $5 \mathrm{~kg}$ (d) five-layered samples due to $10 \mathrm{~kg}$ (e) nine-layered samples due to $10 \mathrm{~kg}$.

It is obvious that the impact energy is in proportion to the height of fall as can be seen in Figure 5. However, the depression does not follow the linear trend. It is mainly owing to the large plastic deformation of Ti sheets caused by impact load, because of little plastic deformation in APC-2 layups. However, in numerical analysis the plastic deformation in Ti sheets was ignored to avoid the complicated nonlinear behavior of simulation.

\section{Conclusion}

Three types of Ti/APC-2 hybrid composite laminates were fabricated. The numerical simulation by using ANSYS LS DYNA-3D software and finite element method was completed to provide the reference information for free drop impact tests and verification by the impact data and failure mechanisms. The equipments of free drop tests were self set up. After all the impact tests the damage samples were subjected to tensile tests to obtain their residual mechanical properties such as the capabilities of loads and compliances. The work can be concluded that in the consideration of friction the height of free drop should be elevated to meet the requirement of equal impact energy in both testing and simulation. The numerical results were more serious damage than those of test data. Thus, the adopted numerical simulation was well acceptable on the conservative side.

\section{Acknowledgements}

The authors would like to acknowledge the Ministry of Science and Technology for the sponsorship under the project MOST 105-2221-E-110-026.

\section{Conflicts of Interest}

The authors declare no conflicts of interest regarding the publication of this paper. 


\section{References}

[1] Ditchek, B.M., Breen, K.R., Sun, T.S. and Venables, J.D. (1980) Morphology and Composition of Titanium Adherends Prepared for Adhesive Bonding. SAMPE P. O. BOX 613 AZUSA CA, 13-24.

[2] Dan-Jumbo, E., Leewood, A.R. and Sun, C.T. (1989) Impact Damage Characteristics of Bismaleimides and Thermoplastic Composite Laminates. Composite Material: Fatigue Fracture ASTM International, 2, 356-317. https://doi.org/10.1520/STP10425S

[3] Lin, C.T., Kao, P.W. and Yang, F.S. (1991) Fatigue Behavior of Carbon Fiber-Reinforced Aluminum Laminates. Composite, 22, 135-141. https://doi.org/10.1016/0010-4361(91)90672-4

[4] Castrodeza, E.M., Ipina, J.E.P. and Bastian, F.L. (2002) Experimental Techniques for Fracture Instability Toughness Determination of Unidirectional Fibre Metal Laminates. Fatigue Fracture Engineering Material Structure, 25, 999-1008. https://doi.org/10.1046/j.1460-2695.2002.00552.x

[5] Vlot, A. (1993) Impact Properties of Fiber Metal Laminates. Composite Engineering, 3, 911-927. https://doi.org/10.1016/0961-9526(93)90001-Z

[6] Vlot, A. (1996) Impact loading on Fibre Metal Laminates. International Journal Impact Engineering, 18, 291-307. https://doi.org/10.1016/0734-743X(96)89050-6

[7] Gunnink, J.W. (1988) Damage Tolerance and Supportability Aspects of Arall Laminate Aircraft Structures. Composite Structure, 10, 83-104. https://doi.org/10.1016/0263-8223(88)90062-1

[8] Khalili, S.M.R., Mittal, R.K. and Kalibar, S.G. (2005) A Study of the Mechanical Properties of Steel/Aluminium/GRP Laminates. Material Science Engineering: A, 12, 137-140. https://doi.org/10.1016/j.msea.2005.08.016

[9] Zhou, Y.X., Wang, Y. and Mallick, P.K. (2004) An Experimental Study on the Tensile Behavior of Kevlar Fiber Reinforced Aluminum Laminates at High Strain Rates. Material Science Engineering. A, 381, 355-362. https://doi.org/10.1016/j.msea.2004.04.027

[10] Jen, M.H.R., Tseng, Y.C. and Li, P.Y. (2007) Fatigue Response of Hybrid Magnesium/Carbon-Fiber/PEEK Nanocomposite Laminates at Elevated Temperature. Journal Japan Society Experimental Mechanics, 7, s56-s60.

[11] Jen, M.H.R., Chang, C.K. and Sung, Y.C. (2015) Fabrication and Mechanical Properties of Ti/APC-2 Hybrid Nanocomposite Laminates at Elevated Temperatures. Journal Composite Material, 50, 2035-2045. https://doi.org/10.1177/0021998315599590

[12] Asundi, A. and Choi, A.Y.N. (1997) Fiber Metal Laminates: An Advanced Material for Future Aircraft. Journal Material Processing Technology, 63, 384-394. https://doi.org/10.1016/S0924-0136(96)02652-0

[13] Dorey, G., Bishop, S.M. and Curtis, P.T. (1985) On the Impact Performance of Carbon Fibre Laminates with Epoxy and PEEK Matrices. Composite Science Technology, 23, 221-237. https://doi.org/10.1016/0266-3538(85)90019-3

[14] Joshi, S.P. and Sun, C.T. (1985) Impact Induced Fracture in a Laminated Composite. Journal Composite Material, 19, 51-66. https://doi.org/10.1177/002199838501900104

[15] Jen, M.H.R., Chang, C.K., Sung, Y.C. and Hsu, F.C. (2010) Fabrication of Ti/APC-2 Nanocomposite Laminates and Their Fatigue Response at Elevated Temperature. 5 th International Conference on Fatigue Composite, Nanjing, October 2010, 317-325. 
[16] Ramani, K., Weidner, W.J. and Kumar, G. (1998) Silicon Sputtering as a Surface Treatment to Titanium Alloy for Bonding with PEKEKK. International Journal Adhesion Adhesives, 18, 401-412. https://doi.org/10.1016/S0143-7496(98)00042-6

[17] Sun, C.T. and Rechak, S. (1988) Effect of Adhesive Layers on Impact Damage in Composite Laminates. In: Composite Materials. Testing and Design (Eighth Conference), ASTM International, 97-1-97-27. https://doi.org/10.1520/STP26130S

[18] Vlot, A. and Gunnink, J.W. (2011) Fibre Metal Laminates: An Introduction. Springer Science \& Business Media.

[19] Wu, C.L., Zhang, M.Q., Rong, M.Z. and Friedrich, K. (2005) Silica Nanoparticles Filled Polypropylene: Effects of Particle Surface Treatment, Matrix Ductility and Particle Species on Mechanical Performance of the Composites. Composite Science Technology, 65, 635-645. https://doi.org/10.1016/j.compscitech.2004.09.004 